\title{
A Numerical Approach Including the Winding Impact for Electrical Machine Vibration Analysis
}

\author{
Limin Huang, Nicolas Galopin, Olivier Chadebec, Gérard Meunier, and Bertrand Bannwarth \\ Univ. Grenoble Alpes, CNRS, Grenoble INP, G2Elab, 38000 Grenoble, France
}

\begin{abstract}
A numerical approach is presented to study and analyze the winding influence on the vibration problem of electrical machines. A multiscale homogenization method is developed to model the mechanical response of the winding structure, which is hard to be finely modeled. Associated with a magneto-mechanical formulation, this approach allows analyzing the mechanical deformation and vibration problem of electrical devices. The computational method has been applied to the magneto-mechanical analysis of a switched reluctance machine. The results show the importance of considering the windings in the vibration analysis of electrical machines.
\end{abstract}

Index Terms - Finite element method, homogenization, magneto-mechanical effects, winding, vibration

\section{INTRODUCTION}

$\mathrm{E}$ LECTRICAL energy in the transport sector is becoming ${ }_{\text {increasingly important as more and more electromagnetic }}$ devices are put into service. However, most electromagnetic devices, particularly electrical machines, can present problems when exposed to high operating constraints and mechanical vibrations. Among the sources of mechanical vibrations, those of electromagnetic origin constitute a complex phenomenon combining electromagnetism and mechanics [1], [2]. Thus, a magneto-mechanical formulation and numerical tools are necessary to study mechanical interactions in electromagnetic devices.

An electromagnetic device can be divided into two parts: an electric excitation part (such as windings) and a magnetic conducting part (such as ferromagnetic core). In the magnetomechanical modeling of electrical devices, the winding is generally not considered in the analysis of deformations or vibrations of the structure due to its topological complexity, meaning that the winding mechanical influence is neglected [3], [4]. However, the winding, due to its elastic properties and mass, directly impacts the mechanical response of the structure. In order to consider the winding influence, especially for solving the mechanical problem, two main methods have been used to date: 1) consider a fine mesh of the heterogeneous winding domain [5] and 2) consider an additional mass of the winding domain [6]. The first method is suitable in the study of high-power machines whose windings are made by copper bars, and in the end-winding modeling. However, for conventional windings made by winding copper wires, this method requires a very fine mesh in the area associated with the winding, which significantly increases the computation time. To deal with the winding modeling problem in the mechanical analysis of electrical machines, the second method equates windings to an increase of the pole mass. Therefore, this method does not allow us to consider the elastic behavior of the winding, which impacts the response of the structure, nor to take into account the forces acting on the winding. This method can only provide a rough evaluation of the winding influence.

To solve the winding modeling problem and analyze its impact, this paper proposes to consider the winding by a homogenized electromagnetic and mechanical behavior. For this purpose, a homogenization method is used to compute the effective mechanical properties of the winding area. Thus, with a magneto-mechanical formulation, the global deformation and the stress distribution can be calculated in both parts (magnetic circuit and windings) of the device. The winding effects on the vibrations of electrical devices are then analyzed by considering the presence of the winding, thanks to its homogenized behavior. Finally, this numerical analysis approach has been applied to the magneto-mechanical analysis of a switched reluctance machine.

\section{Magneto-MechaniCAL Formulation}

To perform the mechanical analysis of electro-technical devices, a classical magneto-mechanical computational procedure based on the finite element method (FEM) is used.

The magneto-mechanical formulation contains three steps, as illustrated in Fig. 1. The first step is to solve the magnetic problem by the finite element method with a classical magnetic vector potential formulation. After the magnetic problem is solved, the second step is to compute the magnetic force distribution by the virtual work method. The nodal magnetic forces associated with mesh nodes are computed from the local derivative of the Jacobian matrix. Once the local magnetic forces are computed, the mechanical problem is solved for the mechanically active parts using a displacement vector formulation.

\section{A. FEM Formulation for Magnetic Problem}

The first step is to solve the magnetic problem. Starting from the magnetostatic phenomenon [7] for an example, the weak formulation of the problem is

$$
\int_{\Omega} v\left(\nabla \times \mathbf{w}_{e}\right) \cdot(\nabla \times \mathbf{A}) \mathrm{d} \Omega=\int_{\Omega_{s}} \mathbf{J} \cdot \mathbf{w}_{e} \mathrm{~d} \Omega
$$

where $\mathbf{A}$ is the magnetic vector potential, $v$ is the inverse of the magnetic permeability of materials, $\mathbf{w}_{e}$ is the test function and $\mathbf{J}$ is the current density. 


\begin{tabular}{|c|c|}
\hline \multicolumn{2}{|c|}{ FEM formulation for solving the magnetic problem } \\
\hline Step 2: & $(\downarrow)$ \\
\hline Magnet & on by the virtual work metho \\
\hline Step 3: & $\downarrow$ \\
\hline
\end{tabular}

Fig. 1. Computation procedure of a magneto-mechanical coupling problem.

The magnetic vector potential $\mathbf{A}$ can be represented as firstorder edge element interpolation [7] as

$$
\mathbf{A}=\sum_{j}^{N_{e}} \mathbf{w}_{e} \cdot A_{j}
$$

where $\mathbf{w}_{e}$ is the test function of edge element $j, A_{j}$ is the tangent component of magnetic vector potential $\mathbf{A}$ on this edge, and $N_{\mathrm{e}}$ is the number of edge elements in the regions mesh. By applying the Galerkin method, the final algebraic solution system is

$$
[\mathbf{R}]\{A\}=\{\mathbf{J}\}
$$

where $[\mathbf{R}]=\sum_{j}\left(\int_{\Omega_{e}} v\left(\nabla \times \mathbf{w}_{e}^{i}\right) \cdot\left(\nabla \times \mathbf{w}_{e}^{j}\right) \mathrm{d} \Omega\right)$, and $\{\mathbf{J}\}$ is the second member associated to the current source and $\{A\}$ is the unknown magnetic vector potential at each DOF.

\section{B. Nodal Magnetic Force Computation}

After the magnetic solution is obtained, the second step is to compute the magnetic force distribution. Among a large number of electromagnetic force calculation methods, the virtual work method can directly calculate the magnetic force distribution at each node of the mesh. Such a method is the most suitable for the following mechanical problem solution. By applying the principle of virtual work method, the nodal magnetic forces associated with mesh nodes are computed from the local derivative of the Jacobian matrix [8]:

$$
\mathbf{f}_{n}=\frac{\partial W}{\partial u} \mathbf{u}=\partial_{u}\left(\int_{\Omega}\left(\int_{0}^{\mathrm{B}} \mathbf{H} \cdot \mathrm{d} \mathbf{B}^{\prime}\right) \mathrm{d} \Omega\right)
$$

where $\partial_{u}$ is a virtual displacement imposed at each node. Assuming the magnetic flux is kept constant, which is achieved by maintaining constant the circulation of the magnetic vector potential on the edges of the element (according to the Stokes' theorem), the magnetic energy $\int_{\Omega_{e}}\left(\int_{0}^{\mathbf{B}} \mathbf{H}\left(\mathbf{B}^{\prime}\right) d \mathbf{B}^{\prime}\right) d \Omega$ can be used to calculate the magnetic force at each node.

\section{FEM Formulation for Mechanical Problem}

Once the local magnetic forces are computed, the mechanical problem is solved for the mechanically active parts using a finite element displacement formulation.

$$
[\mathbf{K}]\{\mathbf{u}\}=\left\{\mathbf{f}_{n}\right\}
$$

where $\mathbf{K}$ is the system stiffness matrix, $\left\{\mathbf{f}_{n}\right\}$ is the nodal

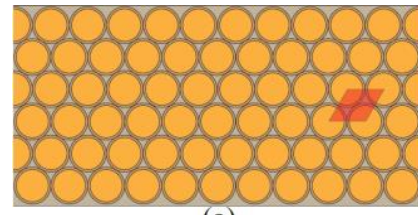

(a)

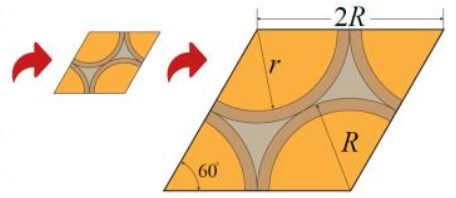

(b)
Fig. 2. Illustration of the multiscale periodic homogenization method: (a) the macroscopic winding area and (b) its microscopic unit cell.

forces which are computed in second step and $\{\mathbf{u}\}$ is the unknown displacement field. The mechanical active parts include magnetic cores and windings.

\section{WINDING HOMOGENIZATION}

From an electromagnetic point of view, the winding homogenization problem is trivial: the winding being traditionally considered as a homogeneous region with an adapted current density. Similar to the electromagnetic homogenization method, the mechanical homogenization is to find out the effective homogeneous properties of the heterogeneous domain.

The periodic homogenization method has been chosen, which presupposes a regularity of the winding. As illustrated in Fig. 2, the principle of the multiscale periodic homogenization method is to select a unit cell that can generate the whole heterogeneous macroscopic domain by repetition [9]. The method consists of solving an elementary problem of elasticity on the unit cell and computing the average of mechanical results to construct the relation between the macroscopic scale and the unit cell. The elasticity problem on the unit cell consists of solving

$$
\left\{\begin{array}{l}
\operatorname{div} \sigma(x)=0 \\
\sigma(x)=\mathbf{c}(x):\left(\varepsilon\left(\mathbf{u}^{*}(x)\right)+\mathbf{E}_{0}\right) \\
\mathbf{u}^{*}(x) \text { in periodic boundary condition }
\end{array}\right.
$$

where the $\sigma$ and $\varepsilon$ are the microscopic stress and strain in the unit cell, and $\mathbf{c}$ is the stiffness matrix of the unit cell. In the elementary problem, $\mathbf{E}_{0}$ is the applied macroscopic strain source, and $\mathbf{u}^{*}$ is the local displacement to be calculated.

Once the elementary problem is solved, the local displacement $\mathbf{u}^{*}$, calculated in relation with a macroscopic deformation applied to the unit cell, allows determining the local strains and stresses. Then, with the averaged microscopic stress $\langle\sigma\rangle$, the global homogenized stiffness matrix $\mathbf{C}^{\text {hom }}$ can be expressed as

$$
\mathbf{C}^{\text {hom }}: \mathbf{E}_{0}=\langle\sigma(x)\rangle=\left\langle\mathbf{c}(x):\left(\varepsilon\left(\mathbf{u}^{*}(x)\right)+\mathbf{E}_{0}\right)\right\rangle
$$

The computational procedure is shown in Fig. 3. For a winding homogenization problem illustrated in Fig. 2, the heterogeneous winding area, consists of three phases: copper conductors, polyimide layers outside the conductors, and epoxy to enhance the insulation ability. After running the 


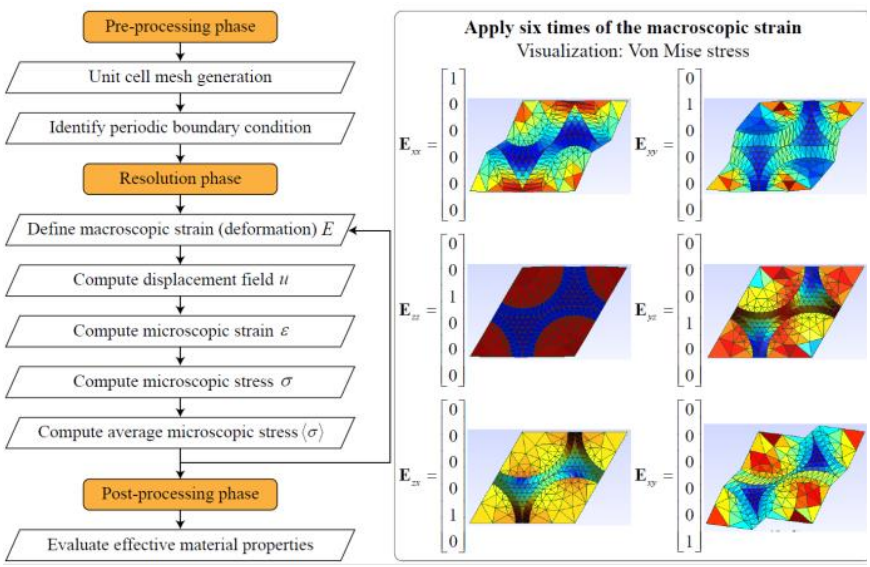

Fig. 3. Computational procedure of the periodic homogenization method.

TABLE I

WINDING HOMOGENIZATION RESULTS

\begin{tabular}{cc|c}
\hline \hline \multicolumn{2}{c|}{ Original heterogeneous winding } & $\begin{array}{c}\text { Homogenized winding } \\
\text { (Orthotopic material) }\end{array}$ \\
\hline Material & Young's Module & $\begin{array}{c}\text { Young's Module } \\
E / \mathrm{GPa}\end{array}$ \\
Copper & $E / \mathrm{GPa}$ & $E_{x x}=10.48$ \\
Polyimide & 120 & $E_{y y}=10.45$ \\
Epoxy & 2.5 & $E_{z z}=62.42$ \\
\hline Material & Poisson's ratio & Poisson's ratio \\
Copper & 0.33 & $v_{x y}=v_{y x}=0.374$ \\
Polyimide & 0.34 & $v_{x z}=v_{y z}=0.055$ \\
Epoxy & 0.34 & $v_{z x}=v_{z y}=0.333$ \\
\hline \multicolumn{2}{c}{} \\
\hline
\end{tabular}

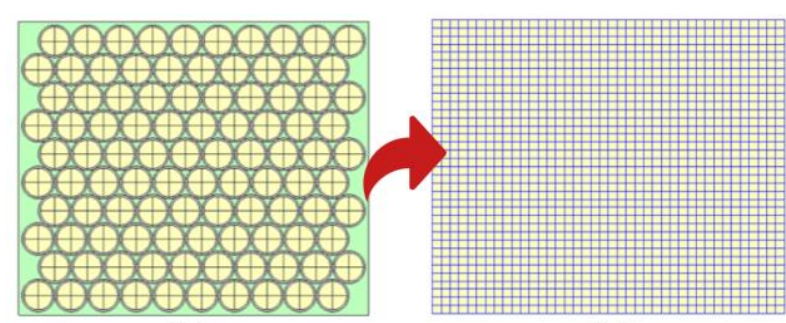

(a)

(b)
Fig. 4. Illustration of the winding domain to be homogenized: a) heterogeneous winding, b) mechanically homogenized winding.

homogenization procedure in the developed computational platform, the effective properties are illustrated in Table I.

To validate the homogenization procedure, the mechanical strain energy is calculated in a heterogeneous winding domain and the corresponding homogenized one. The heterogeneous winding considered is a rectangular domain, composed of 9 unit cells according to the vertical edge and 10 unit cells according to the horizontal edge, as illustrated in Fig. 4. The problem is to impose a displacement on a vertical edge while keeping the second one fixed. Several meshes from coarse to fine have been considered for the homogenized domain.

The differences in the strain energy between the heterogeneous and the homogenized winding domains are presented in Fig. 5. The results show a good agreement of the homogenized procedure, even for a coarse mesh. The $31 \%$ difference is due to border effects where the homogenized properties do not represent well the border of the heterogeneous

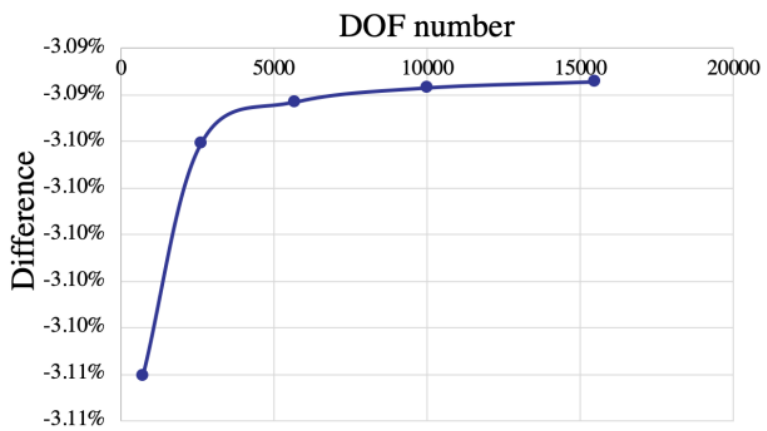

Fig. 5. Winding homogenization convergence results.

medium. For a larger heterogeneous medium, the difference would be much smaller. Moreover, the homogenized domain is robust, and the mesh does not have a significant influence on the results.

\section{Finite Element VibRation Analysis}

To analyze the impact of the winding on the mechanical vibration, responsible for the acoustic noise emission of electric machines, a modal analysis [10] is performed. The method allows evaluating the global displacement of a given node by summing the displacement associated with individuals modes.

Considering an undamped problem, the general equation of motion is given as:

$$
[\mathbf{M}]\{\ddot{\mathbf{u}}\}+[\mathbf{K}]\{\mathbf{u}\}=\{\mathbf{f}\}
$$

with the mass and stiffness matrices defined for each element by:

$$
\begin{aligned}
{\left[M_{e}\right] } & =\iiint_{\Omega_{e}}\left[N_{e}\right] \rho\left[N_{e}\right]^{T} d \Omega \\
{\left[K_{e}\right] } & =\iiint_{\Omega_{e}}\left[B_{e}\right]^{T} \mathbf{C}\left[B_{e}\right] d \Omega
\end{aligned}
$$

where $B_{e}=\partial_{x}\left[N_{e}\right]$ is the strain-displacement matrix, $N_{e}$ is the nodal shape function and $\mathbf{C}$ is the elasticity matrix of element $e$ calculated by the homogenization procedure for the winding.

The free-vibration solutions of the structure, assuming that the solutions have a harmonic shape, lead to the eigensystem:

$$
\left(\mathbf{K}-\lambda_{i} \mathbf{M}\right)\{\mathbf{u}\}_{i}=0
$$

where $\lambda_{i}$ is an eigenvalue associated to eigenvector $\{\mathbf{u}\}_{i}$.

The solution of the equation gives a set of natural frequencies for each vibrational mode, and the associated eigenvectors corresponding to the displacements of each node under a specific vibration mode.

\section{ApPliCATION EXAMPLE: VibRational ANALYSiS OF A SWITCHED RELUCTANCE MACHINE}

The proposed computational approach is applied to a switched reluctance machine (SRM). The SRM has six poles to the stator and four poles to the rotor (rotor diameter $34 \mathrm{~mm}$, stator diameter $64 \mathrm{~mm}$, air gap $0.4 \mathrm{~mm}$, and stator thickness 5 $\mathrm{mm})$. A $2 \mathrm{D}$ resolution of the magneto-mechanical problem is 


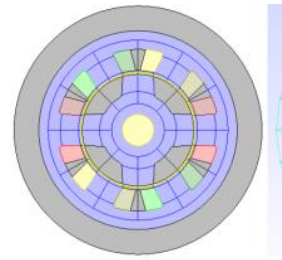

(a)

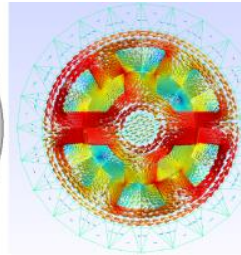

(b)

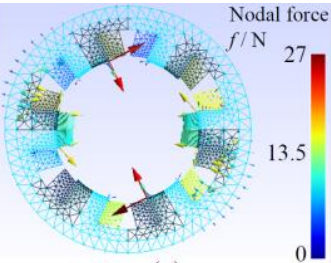

(c)
Fig. 6. Application example of a switched reluctance machine: (a) the geometry, (b) the magnetic flux density distribution, and (c) the nodal magnetic force distribution in the stator (winding included).

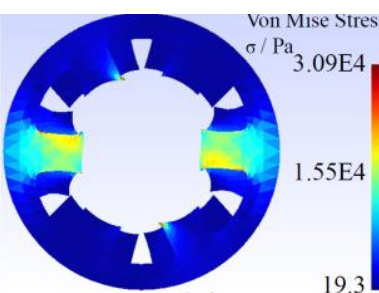

(a)

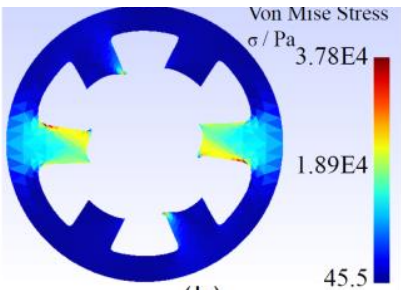

(b)
Fig. 7. Mechanical deformation and stress distribution in stator: (a) considering the homogenized winding and (b) without considering the winding domain.

TABLE II

NATURAL FREQUENCY VALUE COMPARISON

\begin{tabular}{c|c|c|c}
\hline \hline Mode & $\begin{array}{c}\text { With homogenized } \\
\text { winding domain }(\mathrm{Hz})\end{array}$ & $\begin{array}{c}\text { Without winding } \\
\text { domain }(\mathrm{Hz})\end{array}$ & Difference \\
\hline 1 & 1148.967 & 1109.785 & $3.53 \%$ \\
2 & 1947.022 & 1805.582 & $7.83 \%$ \\
3 & 2835.105 & 2951.513 & $-3.94 \%$ \\
4 & 3538.308 & 3553.135 & $-0.42 \%$ \\
5 & 6102.200 & 5630.270 & $8.38 \%$ \\
6 & 7363.029 & 6269.792 & $17.44 \%$ \\
\hline
\end{tabular}

considered, assuming a plane deformation hypothesis. Figure 6 shows the machine geometry, the magnetic flux density distribution, and the local magnetic force distribution in the stator at an operation instant.

The Von Mises equivalent stress is evaluated after solving the mechanical problem on configurations with (Fig. 7a) or without (Fig. 7b) the presence of the windings. A significant difference appears in the distribution and level of equivalent stresses. A difference of $18 \%$ on the average Von Mises equivalent stress is observed between the unwinding configuration ant the winding configuration.

Of course, the winding also affects the vibration modes of the machine structure. Thanks to the homogenization procedure presented in section III, the free-vibration mode values of the SRM chosen structure, with or without winding, are presented in Table II. The results show that for all the six first vibrations modes, a significant difference is obtained on the frequency values. The related mode shapes (Fig. 8) are slightly different when considering the presence of the winding on the stator. This results in differences (between unwinding and winding configurations) on the maximum displacements of the stator outer surface between $27 \%$ and $48 \%$ depending on the deformation modes.

The presence of the winding, therefore, results in a shift in the free-vibration modes and a significant reduction in the associated displacement of the stator outer surface.

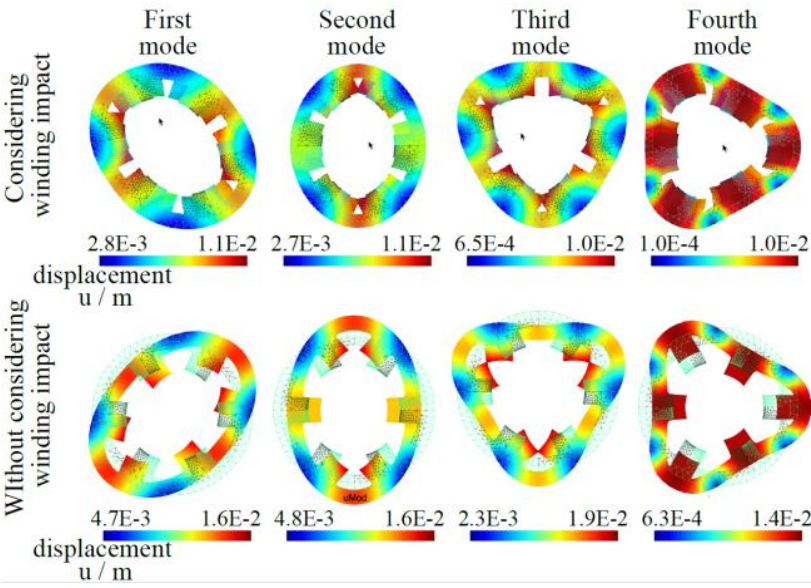

Fig. 8. Vibration mode shapes of the machine at the first four natural frequencies (considering the homogenized winding and without considering the winding domain.

\section{CONCLUSION}

In the paper, a numerical approach for electrical machine vibration analysis is presented to study the winding influence on the mechanical response of electrical machines. The numerical approach is based on a finite element magnetomechanical formulation and a periodic homogenization to define the effective mechanical properties of the winding domain in electrical devices. This approach has been applied to an SRM for which it is clearly highlighted the importance of taking into account the presence of the winding in the vibration analysis of electrical machines, which results in a shift in the frequencies of the deformation modes and a reduction in the amplitudes of the associated deformations.

\section{REFERENCES}

[1] D. Torregrossa, F. Peyraut, B. Fahimi, J. M. Boua, and A. Miraoui, "Multiphysics finite-element modeling for vibration and acoustic analysis of permanent magnet synchronous machine," IEEE Trans. Energy Conversion, vol. 26, no. 2, pp. 490-500, 2011.

[2] A. Tan-Kim, "Vibro-acoustic Simulation and Optimization of a ClawPole Alternator," IEEE Trans. Energy Conversion, Vol. 52 No. 5, pp. 38783885, 2016.

[3] H. Ebrahimi, Y. Gao, H. Dozono, K. Muramatsu, T. Okitsu and D. Matsuhashi, "Effects of stress and magnetostriction on loss and vibration characteristics of motor," IEEE Trans. Magn., vol. 52, no. 3, 8201404, 2016.

[4] L. Zhu, B. Wang, R. Yan, Q. Yang, Y. Yang and X. Zhang, "Electromagnetic vibration of motor core including magnetostriction under different rotation speeds," IEEE Trans. Magn., vol. 52, no. 3, 8102004, 2016.

[5] R. Lin, A. N. Laiho, A. Haavisto, and A. Arkkio, "End-winding vibrations caused by steady-state magnetic forces in an induction machine," IEEE Trans. Magn., vol. 46, no. 7, pp. 2665-2674, 2010.

[6] W. Cai, P. Pillay, and Z. Tang, "Impact of stator windings and endbells on resonant frequencies and mode shapes of switched reluctance motors," IEEE Trans. Magn., vol. 38, no. 4, pp. 1027-1036, 2002.

[7] G, Meunier, The finite element method for electromagnetic modeling, John Wiley \& Sons, 2010.

[8] Z. Ren, and A. Razek "Local force computation in deformable bodies using edge elements," IEEE Trans. Magn., vol. 28, no. 2, pp. 1212-1215, 1992.

[9] J. C. Michel, H. Moulinec, and P. Suquet, "Effective properties of composite materials with periodic microstructure: a computational approach," Computer methods in applied mechanics and engineering, vol. 172, no. 1-4, pp. 109-143, 1999.

[10] K. J. Bathe, Finite Element Procedures, Prentice-Hall, 1996. 